\title{
LETTERS
}

\section{Tackling physician burnout: Too little, too late?}

What is death, and why, as medics, are we so scared of its inevitability? These are difficult questions to answer in a society that desperately strives to preserve life. In a commentary in CMAJ, Drs. Leeat Granek and Sandy Buchman explored the unique insight of palliative care teams in tackling physician burnout. ${ }^{1}$ Health care professionals at all stages of training could benefit from these skills. Intervention must start during medical school.

Medical students have minimal experience with palliative care, with a call coming only recently to deliver consistent education regarding end-of-life care. ${ }^{2}$ We feel that the best way for students to develop skills is to interact with the professionals best equipped with them.
Fortunately, as medical students in our penultimate year at Imperial College London, we are enrolled in the Integrated Clinical Apprenticeship, a scheme that fosters longitudinal learning in the community. ${ }^{3}$ This includes tutorials focused on medical humanities, some led by palliative care physicians, artists and literary experts. In these sessions, topics such as death, fallibility and the role of the doctor are explored. Students are encouraged to reflect on their own emotions regarding these challenging issues. These discussions lay the foundations to develop a culture of compassion and mutual support.

We believe that teaching the next generation of doctors the skills identified by Drs. Granek and Buchman is essential. These "soft" but crucial skills must be nurtured early on, providing resilience to burnout not only for qualified physicians, but throughout education.
Madura Nanthakumaran iBSc

Alice K. Colthurst iBSc

\section{James W. Patrick iBSC}

Medical students

Imperial College London,

London, UK

Cite as: CMAJ 2019 September 3;191:E971. doi: $10.1503 / \mathrm{cmaj} .72562$

\section{References}

1. Granek L, Buchman S. Improving physician wellbeing: lessons from palliative care. CMAJ 2019;191: E380-1.

2. Fitzpatrick D, Heah R, Patten S, et al. Palliative care in undergraduate medical education - How far have we come? Am J Hosp Palliat Care 2017;34: 762-73.

3. Simpkin AL, McKeown AM, Parekh R, et al. A novel Integrated Clinical Apprenticeship: transforming medical students into student doctors. Educ Prim Care 2017;28:288-90.

Competing interests: None declared. 